\title{
The Complexity of the Dependence Operator
}

\section{P. D. Welch}

Received: 2 March 2014 / Accepted: 9 June 2014 / Published online: 4 July 2014

(C) Springer Science+Business Media Dordrecht 2014

\begin{abstract}
We show that Leitgeb's dependence operator of Leitgeb (Journal of Philosophical Logic, 34, 155-192, 2005) is a $\Pi_{1}^{1}$-operator and that this is best possible.
\end{abstract}

Keywords Truth $\cdot$ Dependence $\cdot$ Inductive operator

In [2] Hannes Leitgeb introduced a dependence operator $D^{-1}$ as a relation between sets of sentences in a language $\mathcal{L}_{\mathrm{Tr}}$ such as that for arithmetic (which we shall take here) augmented with a predicate symbol Tr to represent truth. We shall assume the reader has a familiarity with this article and its ideas, as our purpose here is only to correct an error of our own: we had mistakenly persuaded its author that this operator was hyperarithmetic (that is the relation ' $\ulcorner\sigma\urcorner \in D^{-1}(\Phi)$ ' - as a relation of $\sigma$ and $\Phi$ - was $\Delta_{1}^{1}$ ). We had realised that this was false, but unfortunately too late for its suppression in [2]. Since a number of people have subsequently asked about this, we feel we should set the record straight, (and take this opportunity to apologise to Hannes Leitgeb for being so misleading). We thus show here:

Proposition 1 The relation ' $\ulcorner\sigma\urcorner \in D^{-1}(\Phi)$ ' is in general $\Pi_{1}^{1}$ and this is best possible. Indeed if $\Phi_{\alpha}$ is a stage in the increasing monotone hierarchy of dependency sets building up to the least fixed point $\Phi_{\text {lf }}$ of [2] Section 3, then for any $\alpha>0, \Phi_{\alpha}$ is a $\Pi_{1}^{1}$-complete set of gödel numbers.

P. D. Welch $(\bowtie)$

School of Mathematics, University of Bristol, Bristol, England

e-mail: P.Welch@bristol.ac.uk 
Proof The first sentence will follow from the second, so we prove the latter. Recall that we define $\Phi_{0}=\varnothing$ and then:

$$
\begin{gathered}
\Phi_{\alpha+1}=\left\{\ulcorner\varphi\urcorner \mid \varphi \in D^{-1}\left(\Phi_{\alpha}\right)\right\}=\left\{\ulcorner\varphi\urcorner \mid \varphi \text { depends on } \Phi_{\alpha}\right\} \\
=\left\{\ulcorner\varphi\urcorner \mid \forall \Psi\left((\mathbb{N}, \Psi) \models \varphi \leftrightarrow\left(\mathbb{N}, \Psi \cap \Phi_{\alpha}\right) \models \varphi\right)\right\} ; \\
\Phi_{\lambda}=\bigcup_{\alpha<\lambda} \Phi_{\alpha} .
\end{gathered}
$$

Given any $\Pi_{1}^{1}$ set $A \subseteq \mathbb{N}$ we show that for any $\alpha$ that $A$ is (1-1) reducible to $\Phi_{\alpha}$. That is, we show that there is a total (1-1) recursive function $F: \mathbb{N} \longrightarrow \mathbb{N}$ so that $n \in A \leftrightarrow F(n) \in \Phi_{\alpha}$. Indeed there will be a single $F$ that works for all $\alpha$ simultaneously.

By a theorem of Kleene, for any such $\Pi_{1}^{1}$-set $A$ there is a recursive relation $R(u, n) \subseteq \operatorname{Seq} \times \mathbb{N}$ so that:

$$
n \in A \leftrightarrow \forall f \in \mathbb{N}^{N} \exists k_{0} \forall k \geq k_{0}(\neg R(\overline{f\lceil k}, n)) .
$$

Here $\overline{f\lceil k}$ denotes the number in Seq coding the sequence of the first $k$ values of $f$. The idea being that an initial segment $f \uparrow k^{\prime}$ is a sequence, so a node, in a recursive tree (depending on $n$ ) specified by $R$, but $f \uparrow k_{0}$ falls out of the tree, as do all later extensions $f \uparrow k$. See for example the discussion in [4] at 16.4. Then $n \in A$ if and only if the tree is wellfounded and all putative infinite paths $f$ must fall out of the tree at somepoint. Roughly speaking the relation $R(u, n)$ holding indicates that a certain Turing computation has not yet converged and halted; if $\neg R(u, n)$ then from the sequence code $u$, a halting run of computation can be inferred, and indeed this is then naturally so for any sequence code $v$ extending the code $u$ - which we write as $u \subseteq v$. We thus naturally have that $\neg R(u, n) \wedge u \subseteq v \rightarrow \neg R(v, n)$. Hence (1) is also equivalent to

$$
n \in A \leftrightarrow \forall f \in \mathbb{N}^{\mathbb{N}} \exists k_{0}\left(\neg R\left(\overline{f\left\lceil k_{0}\right.}, n\right)\right) .
$$

Here $u \in$ Seq. Finite sequences of numbers such as $\left(u_{0}, u_{1}, \ldots, u_{m-1}\right)$ may be coded by natural numbers $u$ via, usually, some prime power coding: so we may take $u=p_{0}^{u_{0}+1} \cdot p_{1}^{u_{1}+1} \cdot \cdots \cdot p_{m-1}^{u_{m-1}+1}$ where the $p_{i}$ enumerate the primes in ascending order. Since the extension of the predicate $\Psi$ is supposed to be gödel numbers of sentences we adjust our coding by primes to a coding by gödel numbers of selfreferential sentences. The choice here is motivated by the desire that we wish to use sentences, and so gödel codes, that cannot appear in the dependence hierarchy sets $\Phi_{\alpha}$. So let $\lambda$ denote a standard liar sentence. By $\lambda^{u_{0}}$ we mean the $u_{0}$-fold conjunction $(\lambda \wedge \lambda \wedge \cdots \wedge \lambda)$ of $u_{0} \lambda$ 's. We let Seq* be the set of gödel numbers of the form $\left\ulcorner\lambda^{u_{0}+1} \vee \lambda^{u_{1}+1} \vee \cdots \lambda^{u_{m-1}+1}\right\urcorner$ and regard the latter number as also a code for the sequence $\left(u_{0}, u_{1}, \ldots, u_{m-1}\right)$.

Then Seq* is a recursive set of numbers, whilst being disjoint from the set of (codes of) grounded sentences in the sense of [2]. Note that the map ${ }^{*}:$ Seq $\longrightarrow$ Seq* implicitly described above may be assumed recursive. We may for the recursive relation $R(u, k)$ underlying the presentation of $A$ above, then introduce a similar recursive relation $R^{*}(u, k)$ so that $R(u, k) \leftrightarrow R^{*}\left(u^{*}, k\right)$ holds. 
We may thus represent $A$ equivalently by:

$$
n \in A \leftrightarrow \forall f \in \mathbb{N}^{N} \exists k_{0} \forall k \geq k_{0}\left(\neg R^{*}\left((\overline{f\lceil k})^{*}, n\right)\right)
$$

Let $\sigma_{n}$ be the sentence:

$$
\begin{aligned}
& {\left[\exists u \in \operatorname{Seq}^{*} \cap \operatorname{\operatorname {Tr}} \wedge\right.} \\
& \left.\wedge \forall u, v \in \operatorname{Seq}^{*} \cap \operatorname{Tr}\left((u \subseteq v \vee v \subseteq u) \wedge \exists u^{\prime} \in \operatorname{Seq}^{*} \cap \operatorname{Tr}\left(u \subset u^{\prime}\right)\right)\right] \longrightarrow \\
& \longrightarrow \exists u \in \operatorname{Tr} \cap \operatorname{Seq}^{*} \neg \varphi_{R^{*}}(u, \bar{n})
\end{aligned}
$$

(We have written the defining formula for $R^{*}$ as $\varphi_{R^{*}}$.) The antecedent here, when true, guarantees that the set Seq* $\cap \Psi$ forms an unbounded set of initial segments of a function with an infinite domain. The conclusion states that $\Psi$ contains a gödel number $u$ which is a sequence* number witnessing $\neg R^{*}(u, n)$.

Claim: $n \in A \leftrightarrow\left\ulcorner\sigma_{n}\right\urcorner \in \Phi_{1}=\{\ulcorner\varphi\urcorner \mid \forall \Psi((\mathbb{N}, \Psi) \models \varphi \leftrightarrow(\mathbb{N}, \varnothing) \models \varphi)\}$. Hence $\Phi_{1}$ is $\Pi_{1}^{1}$-complete.

Proof Let $n \in A$. Notice that $(\mathbb{N}, \varnothing) \models \sigma_{n}$ trivially, since the extension of $\dot{\text { Tr }}$ is empty and so the antecedent of $\sigma_{n}$ is false, and $\sigma_{n}$ holds vacuously. Now let $\Psi$ be arbitrary; again if $\Psi$ does not contain an infinite linear chain of sequence* numbers, then $\sigma_{n}$ is again vacuously true. However, if otherwise, $\Psi$ will contain arbitrarily long sequence* numbers of initial segments of some function $f: \mathbb{N} \longrightarrow \mathbb{N}$ coded as sequence numbers in Seq*. That is, for some $f \in \mathbb{N}^{N},(\overline{f\lceil k})^{*} \in \Psi$ for infinitely many $k$.

The antecedent of $\sigma_{n}$ now holds true. Because

$$
n \in A \leftrightarrow \forall f \exists k_{0} \forall k \geq k_{0} \neg R^{*}\left((\overline{f\lceil k})^{*}, n\right),
$$

we shall have for some sufficiently large $k$ that $(\overline{f\lceil k})^{*}$ both witnesses that the consequent of $\sigma_{n}$ holds and is in $\Psi$. Thence $\left\ulcorner\sigma_{n}\right\urcorner \in \Phi_{1}$.

However if $n \notin A$ then by the properties of $R$ and $R^{*}$ discussed above, we have

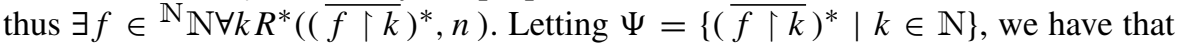
$(\mathbb{N}, \Psi) \models \neg \sigma_{n} \wedge(\mathbb{N}, \varnothing) \models \sigma_{n}$. Hence $\left\ulcorner\sigma_{n}\right\urcorner \notin \Phi_{1}$. The Claim is proven.

Setting $F(n)=\left\ulcorner\sigma_{n}\right\urcorner, F$ is recursive and witnesses the required reduction. Now notice that by our choice of sequence* numbers, none of these can be in any $\Phi_{\alpha}$. Hence the argument above equally shows directly that $n \in A \leftrightarrow F(n)=\left\ulcorner\sigma_{n}\right\urcorner \in \Phi_{\alpha}$. This proves the proposition.

The successive steps of this operator when started at $\Phi_{0}=\varnothing$ yield at each and every stage $\Pi_{1}^{1}$-complete sets of integers. And then after $\omega_{1}^{\mathrm{ck}}$ many steps the resulting fixed point $\Phi_{\mathrm{lf}}=\Phi_{\omega_{1}^{\text {ck }}}$ is also $\Pi_{1}^{1}$-complete. Very similar arguments show that the same is true for the supervaluation operator $\Gamma^{\mathrm{sv}}$ : starting out from $\Gamma_{0}=\varnothing$ yields that all the $\Gamma_{\alpha}$ are $\Pi_{1}^{1}$-complete sets (this was observed by Greg Hjorth and Toby Meadows). In this case it does not matter which mode of supervaluation one takes, 
whether it be via maximal consistent extensions of a pair $\left(\Gamma_{\alpha}^{+}, \Gamma_{\alpha}^{-}\right)$, or simply all consistent extensions, or ..., just as for the dependence operator the universal quantification over all relevant extensions, is a universal quantification over all countable sets. This is in contradistinction to the hierarchy formed by using the strong (or the weak) Kleene truth tables to formulate extensions. In these cases a $\Pi_{1}^{1}$-complete set is only attained at the final union at stage $\omega_{1}^{\mathrm{ck}}$. To build a new stage $\Gamma_{\alpha+1}^{\mathrm{sK}}$, impeccable evidence using strong Kleene logic from the data in $\Gamma_{\alpha}^{\mathrm{sK}}$ is used; it is an incrementalist approach. (The transitional operator at each stage is low down in the hyperarithmetic hierarchy being just beyond arithmetic; the earlier stages are then all hyperarithmetic.) Whereas for the dependence and the supervaluation operators, a more cavalier, but ultimately a completist, approach is used that casts its eye wide over all conceivable extensions, to obtain a still reasonable, but as full as possible an extension in one swoop, which however will still need revising upwards. So the conceptions (and attitudes) of course, as we know, are quite different. One might wonder why the supposed universal quantifier over all functions, or all sets, in the supervaluation and dependence construction is not a $\Pi_{1}$ quantification over the real continuum, or equivalently over all (hereditarily) countable sets? This would render such a quantification expressed by a $\Pi_{2}^{1}$ statement. This is because, to determine whether $\ulcorner\sigma\urcorner$ is in the next extension, despite the prima facie supervaluation quantification implicit in $\Gamma^{\mathrm{sv}}$ being over all possible extensions, one really only needs to look at all possible extensions in the next admissible set (that is, transitive model of Kripke-Platek set theory) beyond $\omega_{1}^{\mathrm{ck}}$. Thus the quantification is really (but implicitly) a bounded universal quantification. (The reason for this pleasantly bounded state of affairs is the Kleene Basis Theorem (see, eg., again Rogers [4], Theorem XLII), which in our context would state that a counterexample to $\ulcorner\sigma\urcorner$ being in the next extension, if such exists at all, could be found recursively in a $\Pi_{1}^{1}$-complete set $P$ and hence would in the least admissible set containing $P$ as an element. Hence to check $\ulcorner\sigma\urcorner$ 's status we need only look there.) However the full-blooded revision theory of Gupta and Belnap [1]which requires considering all possible revision sequences and all possible revision rules etc.,etc., over the natural number model, necessarily requires an unbounded quantification over the real continuum to determine their stable truth set, which is then indeed a complete $\Pi_{2}^{1}$ set.

Dependence is, by its nature, also a notion that requires one to look around at all possible extensions, and so it is perhaps unsurprising that a somewhat complicated (meaning a complete $\Pi_{1}^{1}$ ) set of outcomes occurs at each stage. This phenomenon is observable again in other presentations of the operators and fixed points, either via games [5] or, reinterpreting these once more, as proof trees [3].

\section{References}

1. Gupta, A., \& Belnap, N. (1993) The revision theory of truth. MIT Press.

2. Leitgeb, H. (2005). What truth depends on. Journal of Philosophical Logic, 34, 155-192.

3. Meadows, T. (2014). Proofs for semantic truth, submitted.

4. Rogers, H. (1967). Recursive Function Theory. Higher Mathematics. McGraw.

5. Welch, P.D. (2009). Games for truth. Bulletin of Symbolic Logic, 15(4), 410-427. 